\title{
LYAPUNOV TYPE INEQUALITY FOR THE EQUATION INCLUDING 1-dim $p$-LAPLACIAN
}

\section{KOHTARO WATANABE}

Abstract. Lyapunov type inequality, for the existence of the solution of the equation including (generalized) $p$-Laplacian:

$$
(-1)^{(m)}\left(\left|u^{(m)}(x)\right|^{p-2} u^{(m)}(x)\right)^{(m)}=r(x)|u(x)|^{p-2} u(x) \quad(a \leqslant x \leqslant b)
$$

under clamped boundary condition is obtained. The usage of the best constant of $L^{p}$ Sobolev inequality clarifies the process for obtaining such inequality.

Mathematics subject classification (2010): 34B27, 46E35.

Keywords and phrases: $p$-Laplacian, Lyapunov inequality, Sobolev inequality, best constant.

\section{REFERENCES}

[1] R. Brown And D. Hinton, Lyapunov inequalities and their applications, Surveys on classical inequalities, 2000, Kluwer Academic Publisher.

[2] C. HA, Eigenvalues of a Strum-Liouville problem and inequalities of Lyapunov type, Proc. of A.M.S. 126 (1998), 3507-3511.

[3] J. P. PINASCO, Lower bounds for eigenvalues of the one-dimensional p-Laplacian, Abst. Appl. Analysis 2004, 2 (2004), 147-153.

[4] J. P. Pinasco, Comparison of eigenvalues for p-Laplacian with integral inequalities, Appl. Math. and Computation 182 (2006), 1399-1404.

[5] K. Watanabe, Y. Kametaka, A. Nagai, H. Yamagishi, K. Takemura, Symmetrization of functions and the best constant of 1-dim $L^{p}$ Sobolev inequality, J. Inequal. and Appl. 2009 (2009), Article ID 874631.

[6] X. YAnG, On inequalities of Lyapunov type, Appl. Math. and Comp. 134 (2003), 293-300. 